\title{
Clinical evaluation of concussion: the evolving role of oculomotor assessments
}

\author{
Eric S. Sussman, MD, Allen L. Ho, MD, Arjun V. Pendharkar, MD, and Jamshid Ghajar, MD, PhD \\ Department of Neurosurgery, Stanford University School of Medicine, Stanford, California
}

Sports-related concussion is a change in brain function following a direct or an indirect force to the head, identified in awake individuals and accounting for a considerable proportion of mild traumatic brain injury. Although the neurological signs and symptoms of concussion can be subtle and transient, there can be persistent sequelae, such as impaired attention and balance, that make affected patients particularly vulnerable to further injury. Currently, there is no accepted definition or diagnostic criteria for concussion, and there is no single assessment that is accepted as capable of identifying all patients with concussion. In this paper, the authors review the available screening tools for concussion, with particular emphasis on the role of visual function testing. In particular, they discuss the oculomotor assessment tools that are being investigated in the setting of concussion screening.

http://thejns.org/doi/abs/10.3171/2016.1.FOCUS15610

KEY WORDS concussion screening; concussion diagnosis; vision tracking; eye tracking; oculomotor tracking; traumatic brain injury

$\mathrm{T}$ RAUMATIC brain injury (TBI) is a major cause of morbidity and mortality throughout the world. ${ }^{22}$ The majority of these injuries are associated with a Glasgow Coma Scale (GCS) score of 13-15 and are classified as mild TBIs (mTBIs). ${ }^{25}$ Sports-related concussion leads to a change in brain function following a direct or an indirect force to the head, is identified in awake individuals, ${ }^{7}$ and represents a considerable proportion of mTBIs. ${ }^{38}$ Despite this classification as "mild," however, concussions are far from benign. Concussed patients frequently suffer from short-term, nonspecific neurological symptoms including headache, fatigue, irritability, photosensitivity, confusion, and difficulty with memory, speech, and/ or sleep. In some patients, these complaints can persist in the so-called postconcussion syndrome. ${ }^{31,44}$ Furthermore, a growing body of scientific research demonstrates substantially higher rates of depression and dementia in the concussed patient population. ${ }^{21,32,42}$

In the United States alone, an estimated 3.8 million sports-related concussions occur every year, ${ }^{30,32}$ and this number is probably an underestimate as it was extrapolated from emergency room visits and does not account for concussions treated in the nonemergent setting. Furthermore, a significant number of patients with concussion never present for medical attention.
Although the neurological signs and symptoms of concussion can be subtle and transient, there are persistent subclinical sequelae, such as impaired attention and balance, that make affected patients particularly vulnerable to further injury. Concussed individuals are 3 times more likely to sustain a recurrent concussion in the same athletic season, ${ }^{23}$ and the force required to cause a recurrent concussion may be less than that required to cause such an injury in a never-concussed individual. ${ }^{4,8}$ Furthermore, previously concussed patients are possibly at risk for a rare but devastating disorder known as "second-impact syndrome." In this syndrome, which can follow a recurrent injury to an incompletely recovered brain, dysfunctional vascular autoregulatory mechanisms following an initial injury lead to vascular engorgement and increased cerebral blood volume that can precipitate diffuse cerebral edema. ${ }^{8}$ Therefore, it follows that the reliable and timely detection of concussion must be a priority in athletics of every level.

In this paper, we review the available screening tools for concussion, with particular emphasis on the role of visual function testing. In particular, we discuss the oculomotor assessment tools that are being investigated in the setting of concussion screening.

ABBREVIATIONS BESS = Balance Error Scoring System; DVS = dynamic visuo-motor synchronization; KDT = King-Devick Test; mTBI = mild traumatic brain injury; SAC = Standardized Assessment of Concussion; SCAT-3 = Sport Concussion Assessment Tool, 3rd Edition; TBI = traumatic brain injury; TGT = tandem gait test. 


\section{Screening Tests for Concussion}

Concussion most often occurs following blunt force injury to the head, which results in shear-induced diffuse axonal stretching due to differential acceleration of the brain relative to the cranial vault, and affects mainly anterior brain structures given their position farthest away from the neck's axis of rotation. Because of the diffuse nature of this neuronal injury, concussion can affect any of a broad range of neurological functions; however, the main functions affected can be broadly categorized as attention and vestibular balance. The array of screening assessments for concussion primarily screens for abnormalities in these domains.

At present, no single assessment is accepted as capable of identifying all patients with concussion. Thus, while the concussion research community continues its endeavor to develop a screening tool with $100 \%$ sensitivity and specificity, additional efforts must focus on identifying an optimal combination of existing screening tools and neurological assessments to best serve athletes here and now.

\section{Symptom Checklists}

Perhaps the most simple and straightforward way to screen for concussion is to interrogate an individual for relevant symptoms following a witnessed or reported traumatic force to the head. According to one study, the most common symptoms include headache (93\%), dizziness (75\%), difficulty with concentration (57\%), confusion (46\%), and visual disturbance or photosensitivity (38\%). ${ }^{39}$ A substantial proportion of patients also reported nausea, drowsiness, amnesia, irritability, and feeling as if in a "fog" or dazed. Importantly, none of these symptoms, either alone or in combination, is specific for concussion, and oftentimes concussions can be undetectable by symptom screening alone. Furthermore, symptom reporting is subjective and can be misrepresented by athletes attempting to evade detection to remain in play. ${ }^{53}$

\section{Standardized Assessment of Concussion}

The Standardized Assessment of Concussion (SAC) is a brief cognitive test that specifically evaluates orientation, concentration, and memory. While the test is easy to administer as a sideline screening tool, it suffers from inadequate sensitivity to justify its use as a stand-alone test. ${ }^{55}$ Furthermore, as with symptom checklists, determined athletes can manipulate the outcome, either by memorizing certain portions of the evaluation ${ }^{15}$ or by intentionally underperforming in the preseason baseline assessment to which subsequent tests will be compared.

\section{Balance and Gait Assessments}

Both balance and gait can also be affected in the setting of concussion, and numerous sideline assessments are intended to evaluate these sensorimotor functions. The Balance Error Scoring System (BESS) is a static balance assessment that requires an individual to perform 3 stances on 2 different surfaces for a total of 6 trials. Each trial is 20 seconds in duration, and the score is equal to the cumulative number of balance errors. ${ }^{1}$ While balance itself is a relatively objective measure of sensorimotor function, significant variability in scoring is reflected by poor interrater and even intrarater reliability ${ }^{16}$ Furthermore, an individual's score on the BESS can fluctuate during the course of an athletic season independent of concussion status, ${ }^{6}$ and the BESS score can be further confounded by lower-extremity injuries and/or fatigue. ${ }^{14,56}$ The timed tandem gait test (TGT), on the other hand, is a dynamic assessment of sensorimotor function in which a participant is timed while walking heel-to-toe along a $38-\mathrm{mm}$ wide piece of tape that is $3 \mathrm{~m}$ in length. Each assessment consists of 4 identical trials, and the best time among the 4 trials is recorded as the official score. ${ }^{19}$ Timed TGT performance can also be affected by exercise and fatigue but less so than with the BESS. ${ }^{26,50}$

Recently, attention has turned to more objective sensorimotor assessment modalities, including portable inertia sensors and the Nintendo Wii Balance Board. While initial studies have been promising, ${ }^{9,28}$ further clinical validation is necessary before these tests can be reliably used for concussion screening.

\section{Sport Concussion Assessment Tool, 3rd Edition}

The Sport Concussion Assessment Tool, 3rd Edition (SCAT-3) consists of a carefully selected series of tests, including a focused physical exam, a 22-symptom checklist, the GCS, and cognitive and sensorimotor assessments.38 More specifically, cognition is assessed with the SAC, and the BESS and/or TGT are used to evaluate sensorimotor function. The SCAT-3 benefits from its ability to assess a range of neurological functions, including orientation, cognition, memory, balance, and gait. However, the duration of the test battery is approximately 15-20 minutes, which is not optimal in the setting of time-limited athletic competition. Furthermore, the SCAT-3 is designed to be administered by medical practitioners, which limits its utility in youth and high-school sports, in which medical professionals are not necessarily available for sideline concussion screening. Similar to many of the other concussion screening tools discussed above, the SCAT-3 also requires baseline testing for comparison, which carries additional logistical challenges. Finally, SCAT-3 is not $100 \%$ sensitive for identifying patients with concussion, ${ }^{32,54}$ thus relegating it to the role of a complementary test rather than the primary stand-alone tool for concussion detection.

\section{King-Devick Test}

Vision is another neurological function that can be assessed as a component of concussion screening. The King-Devick Test (KDT) is a rapid number-naming task in which an individual reads aloud 3 cards of irregularly spaced single-digit numbers as quickly as possible. The total score is equal to the sum of the times required to read through each card (Fig. 1). At its core, the KDT is an assessment of visual function, but it also assesses the integrity of attention. ${ }^{41}$ Similar to the tests discussed above, the KDT requires a baseline assessment for comparison. Healthy athletes have been shown to improve on the KDT with repeated testing ${ }^{18}$ and with exercise. ${ }^{18,41}$ Thus, any decline in performance (that is, an increase in score time) compared with baseline testing suggests concussion..$^{17,18,20,27}$ In 


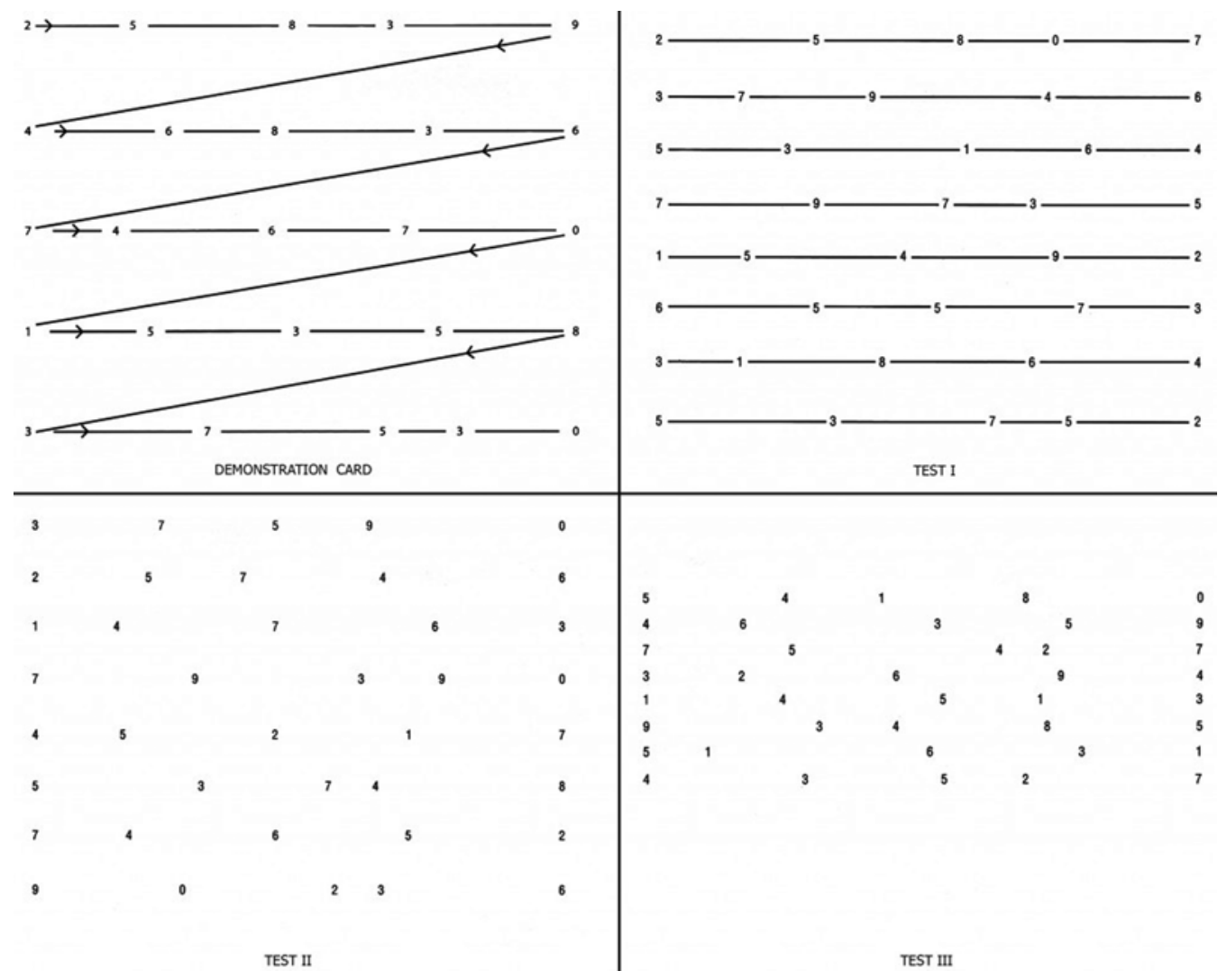

FIG. 1. King-Devick Test, which consists of 1 demonstration card and 3 test cards. Participants are instructed to read the numbers on each card from left to right as quickly as possible without making any errors. The sum of the time scores from all 3 test cards is the summary score or K-D time score for the entire test. The number of errors made in reading the test cards is also recorded. Reproduced from Leong et al: J Optom 8:131-139, 2015. CC BY-NC-ND 4.0. http://creativecommons.org/licenses/by-nc-nd/4.0/.

the setting of sideline concussion screening, the KDT is ideal in that it takes less than 1-2 minutes to complete and can be administered by nonmedical personnel such as parents or coaches. However, the KDT is not $100 \%$ sensitive for detecting concussion and thus should not be used as a stand-alone test. One study demonstrated that the KDT was more sensitive than either the TGT or the SAC alone, but it still had only $75 \%$ sensitivity for detecting concussed athletes..$^{19}$ Instead, current evidence suggests that the KDT can serve as a complementary test to increase the sensitivity of combinations of the previously discussed screening tools. More specifically, the addition of the KDT to a sideline concussion screening protocol consisting of SAC and TGT was superior to SAC or TGT alone. ${ }^{19}$ In a separate study, the combination of SAC and BESS, as used in the SCAT-3, had 90\% sensitivity for concussion, and this rate increased to $100 \%$ with the addition of the KDT. ${ }^{54}$

\section{Oculomotor Assessment for Concussion Screening \\ Rationale}

As noted above, concussion screening has historically focused on a broad range of neurological functions, from cognition to balance. However, a key symptom of both the immediate and delayed postinjury effects of concussion is attention impairment. ${ }^{2,3}$ Monitoring the attentional states of patients is important for concussion diagnosis, as well as for assessing recovery and prevention of recurrent injury. In fact, eye-tracking protocols have been used in the assessment of postconcussive patients with residual symptoms and have identified persistent objective abnormalities in eye movements that correlate to the severity of postconcussive syndromes. ${ }^{10,24}$ The overlap between gaze and attention networks has been demonstrated utilizing functional MRI. ${ }^{12}$ Thus, more recently, visual testing has been increasingly recognized as a sensitive tool in the evaluation of this patient population, as has the KDT described above. Approximately half of the neural connections in the human brain-including cortical, subcortical, cerebellar, and brainstem networks - are involved in visual function. Given the diffuse and nonspecific nature of neurological injury in concussion, it follows that the integrity of a diffuse neuronal network- such as that related to visual function-can be affected. In fact, some form of visual dysfunction occurs in up to $90 \%$ of concussed patients. ${ }^{11,25}$

Vision and oculomotor function, in particular, have been targeted as practical and reliable surrogates for attention in the diagnosis of concussion. There are several different classes of eye movements, and a basic understanding of these is essential to understand the foundation of oculomotor testing in the setting of suspected concussion. "Visual fixation" holds an image in a constant position on the retina while the head is stationary. Fixation is not con- 
sidered a movement but is under active neural control. No studies have demonstrated differences in measures of fixation among concussion patients. ${ }^{5,10}$ A "saccade" is a rapid change in the orientation of the eye and can aid in bringing an object back into focus in the eye. Saccades are a key component of the KDT discussed above ${ }^{18,27}$ and can also be assessed utilizing eye trackers, ${ }^{10,13}$ head-mounted saccadometers ${ }^{40}$ or electrooculography. ${ }^{29}$ "Smooth pursuit" helps stabilize the image of a moving target in the eye, and changes in smooth pursuit that have been identified in concussion patients include decreased target position, increased eye position error, low velocity gains, and uneven gaze trajectory with large saccadic jumps ahead of the target. $37,51,52$ "Vergence" is the simultaneous movement of both eyes in opposite directions to maintain single binocular vision. Deficits in near-point convergence have been identified in several studies of concussion patients..$^{24,43}$ While there are a number of other types of eye movements and ocular reflexes, the ones listed above serve to stabilize and support foveal vision and are thus purposeful in nature. "Visual tracking" is a combination of smooth pursuit and saccadic eye movements that allows for stability and continuous observation of targets in motion. This provides an ideal model for studying the advanced neurological function of anticipating the temporal course and trajectory of a stimulus. Combined tracking of the pupil and corneal reflection allows for very precise measurement of visual tracking and has been validated as a biomarker for anticipatory neural activity. ${ }^{35-37}$

\section{Visual Tracking}

Several studies have utilized video-oculography techniques and devices to evaluate visual tracking in the context of concussion diagnosis and management. Tracking of a circular target trajectory is particularly well suited for measuring predictive visual tracking given its highly predictable trajectory and quantification with simple parameters such as smooth pursuit velocity gain, phase error, root-mean-square error, and gaze position error variability (Fig. 2). ${ }^{34}$ Maruta et al. used video-oculography to identify significant changes in visual tracking in patients with postconcussive syndrome. ${ }^{37}$ These changes were correlated with evidence of white matter tract damage in the corona radiata and genu of the corpus callosum on diffusion tensor imaging, as well as declines in attention and working memory on neurocognitive testing. This same group has also used the EYE-SYNC system, which quantifies the predictive timing of dynamic visuo-motor synchronization (DVS) between gaze and target during predictive circular visual tracking. They characterized normative and longitudinal data for the system for normal individuals and demonstrated a significant decline in DVS scores among a small cohort studied within 2 weeks of concussive injury (Fig. 3). These DVS scores did eventually recover to normal range. ${ }^{35}$ Samadani et al. recently used a nonspatially calibrated, binocular, eye movement-tracking algorithm to detect dysconjugate gaze in concussion patients relative to that in healthy controls. ${ }^{49}$ Measures of horizontal dysconjugacy were significantly increased in concussion patients and correlated with SCAT-3 and SAC scores. These same metrics also improved back to

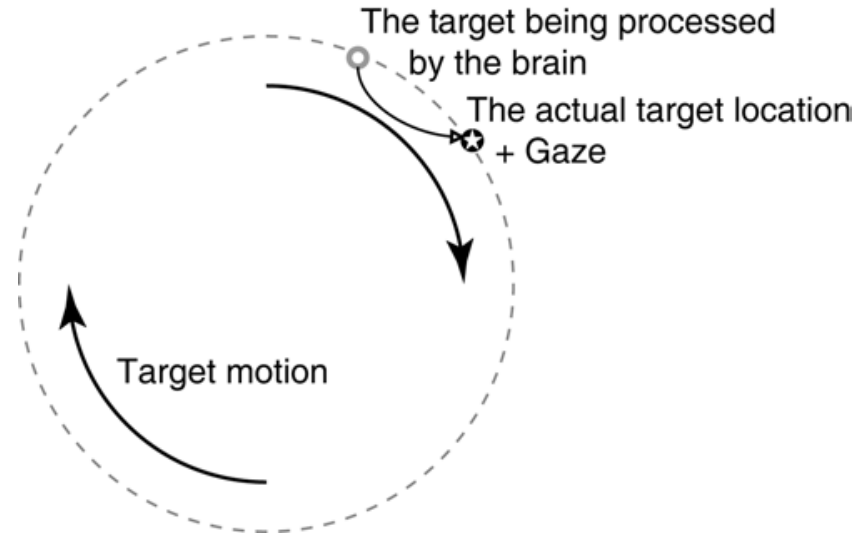

FIG. 2. Prediction visual tracking with circular target trajectory. Maintaining gaze on a circular target trajectory requires synchronization of the motor output of the eye with a predicted target motion rather than any incoming sensory information. Reproduced from Maruta and Ghajar: Prog Neurol Surg 28:226-233, 2014. Copyright @ 2014 Karger Publishers, Basel, Switzerland.

baseline at follow-up testing. This novel eye-tracking algorithm seeks to overcome some of the limitations of previous spatially calibrated eye-tracking techniques but has been questioned by some investigators. ${ }^{33,46,47}$ In a subsequent expanded study utilizing the same methodology, Samadani et al. demonstrated an $88 \%$ sensitivity and $87 \%$ specificity for this eye-tracking technology ${ }^{48}$

\section{Discussion}

Recent research has identified a multitude of previously unrecognized short- and long-term health consequences of sports-related concussion, bringing this subtype of mTBI to the forefront of medical research and even national media attention. In particular, it is now clear that the occurrence of a single concussion can lower the threshold for subsequent concussions-especially in the short term probably because of persistent impairments in attention and/or balance. Thus, there has been an urgent effort to improve the reliability of concussion screening so that affected individuals can be appropriately removed from competition until it is deemed safe to return to play.

When evaluating the utility of concussion tests, it is important to distinguish between sideline screening assessments and evaluations that are more suitable for definitive diagnosis or monitoring of postconcussive symptoms. In this paper we reviewed the most commonly employed sideline concussion screening tools, including symptom checklists, the SAC, the BESS, the timed TGT, the SCAT-3, and the KDT, and discussed the emerging role and strategies for oculomotor assessment in suspected concussion patients. Although the topic is beyond the scope of this paper, it is important to recognize that the armamentarium for concussion testing extends beyond the above-mentioned sideline screening tools and includes comprehensive computerized neurocognitive assessments, serum biomarkers, neuroimaging, and electrophysiological studies.

It is also important to note that subconcussive forces to the head can result in clinically significant symptoms 

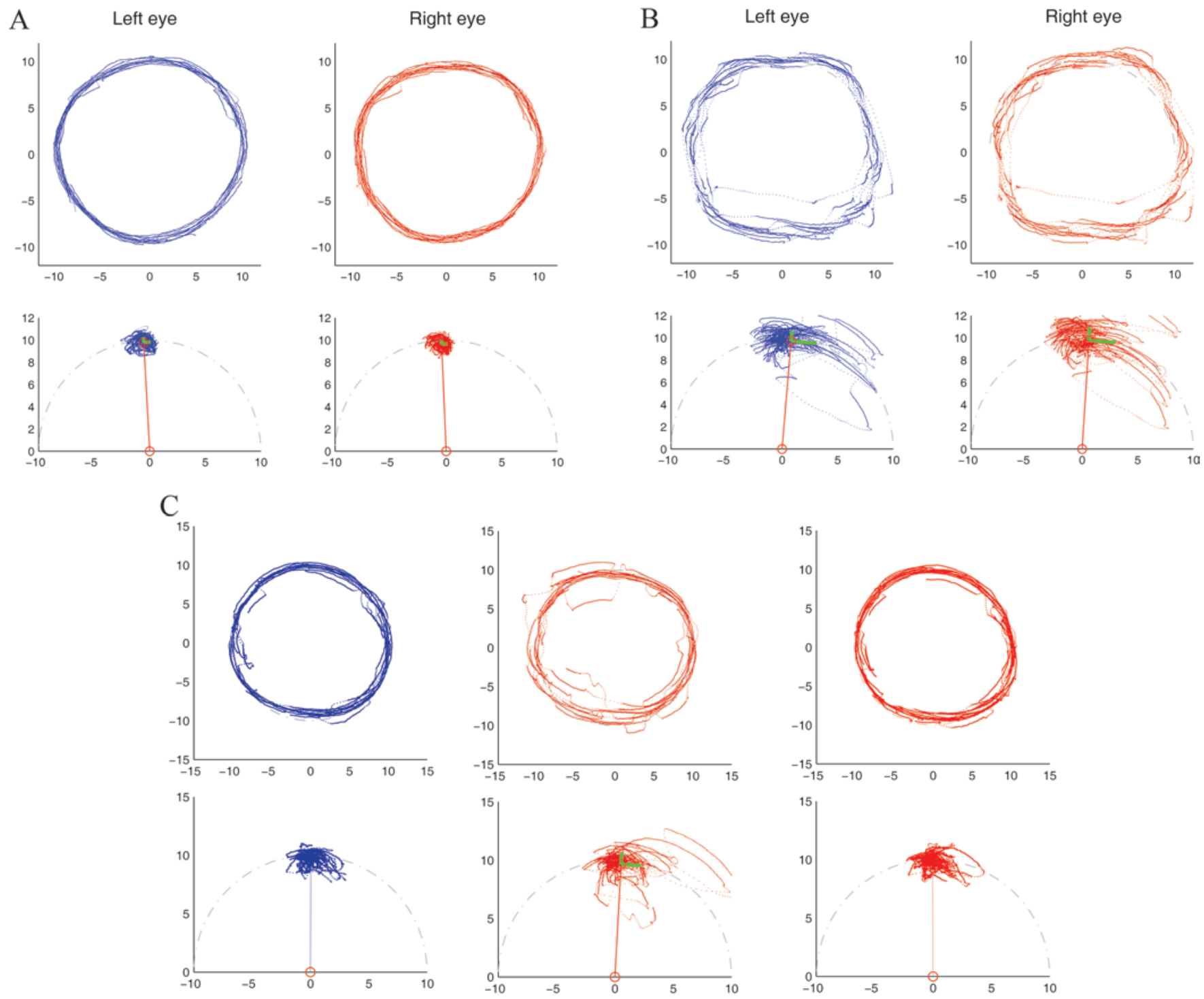

FIG. 3. Representative scattergrams of gaze positions (blue indicates left eye, and red indicates right eye) relative to the target; gaze positions were gathered at a frequency of $500 \mathrm{~Hz}$. Circular patterns represent the path of the eye following a dot moving in a circle, and the semicircular pattern represents the eye position versus the target. Deviation from a target trajectory (dashed line) in both normal (A) and postconcussive (B) patients. A concussion signal is indicated by eye positions jumping ahead of the dot shown in $B$ and $C$. From left to right (C), patient data at baseline, immediately postconcussion, and 2 days postconcussion.

that are not widely recognized or tested for in athletic competition. These injuries do not typically result in the characteristic signs and symptoms of true concussive injuries; however, there is a growing body of evidence that subconcussive injuries do have a subtle but cumulatively significant impact on neurophysiological functions. ${ }^{45}$ Prior research has suggested that the severity of oculokinetic dysfunction correlates with the severity of neurological dysfunction in concussion. ${ }^{24}$ To the extent that subconcussive injuries can represent a milder injury on the concussion spectrum, one can speculate that such injuries may result in objectively similar but subtler abnormalities on visual tracking assessments. Oculomotor testing has proven to be a sensitive technique for detecting subtle disruptions in neural function and should be further investigated in the context of subconcussive head injury.
The concussed population is unique in that individuals are typically young, physically able, and otherwise healthy. Thus, it is particularly important for any sideline screening tool to be highly sensitive because the consequences of failing to identify a concussed patient can be potentially catastrophic. Simultaneously, in the competitive setting of athletics-where there is a demand to consistently participate and perform at a high level - an effective concussion screening tool must be quick and highly specific. Ideally, a sideline screening assessment should also be standardized, cost-effective, and able to be administered by nonmedical personnel, such that it is practical in youth and high-school athletics, as well as collegiate and professional sports. In addition, as discussed above, intentional manipulation of preseason baseline testing is a ubiquitous problem with concussion screening tools, thus 
underscoring the need for the development of normative, age-based data sets that would eliminate the need for baseline assessments.

At present, a $100 \%$ sensitive and specific concussion screening assessment does not exist, and it is unreasonable to expect that such a flawless tool can be developed. Given the diffuse nature of neurological injury in sportsrelated concussion, an effective screening protocol must assess a range of neurological functions, and this will be achieved using a combination of assessments. Further investigation is necessary to determine the precise combination of screening tools that has the highest sensitivity and specificity for detecting concussion. Moreover, future research should aim to further clarify the role of oculomotor assessments in concussion screening and to optimize the assessment of this neurological function for sideline concussion screening. Finally, it is essential to remember the importance of concussion education ${ }^{57}$ and prevention and concussion screening as parallel solutions to this complex problem.

\section{References}

1. Bell DR, Guskiewicz KM, Clark MA, Padua DA: Systematic review of the balance error scoring system. Sports Health 3:287-295, 2011

2. Bernstein DM: Recovery from mild head injury. Brain Inj 13:151-172, 2009

3. Binder LM, Rohling ML, Larrabee GJ: A review of mild head trauma. Part I: Meta-analytic review of neuropsychological studies. J Clin Exp Neuropsychol 19:421-431, 1997

4. Boden BP, Tacchetti RL, Cantu RC, Knowles SB, Mueller FO: Catastrophic head injuries in high school and college football players. Am J Sports Med 35:1075-1081, 2007

5. Brahm KD, Wilgenburg HM, Kirby J, Ingalla S, Chang CY, Goodrich GL: Visual impairment and dysfunction in combatinjured servicemembers with traumatic brain injury. Optom Vis Sci 86:817-825, 2009

6. Burk JM, Munkasy BA, Joyner AB, Buckley TA: Balance error scoring system performance changes after a competitive athletic season. Clin J Sport Med 23:312-317, 2013

7. Carney N, Ghajar J, Jagoda A, Bedrick S, Davis-O'Reilly C, $\mathrm{du}$ Coudray H, et al: Concussion guidelines step 1: systematic review of prevalent indicators. Neurosurgery 75 (Suppl 1):S3-S15, 2014

8. Centers for Disease Control and Prevention (CDC): Sportsrelated recurrent brain injuries-United States. MMWR Morb Mortal Wkly Rep 46:224-227, 1997

9. Chang JO, Levy SS, Seay SW, Goble DJ: An alternative to the balance error scoring system: using a low-cost balance board to improve the validity/reliability of sports-related concussion balance testing. Clin J Sport Med 24:256-262, 2014

10. Cifu DX, Wares JR, Hoke KW, Wetzel PA, Gitchel G, Carne W: Differential eye movements in mild traumatic brain injury versus normal controls. J Head Trauma Rehabil 30:21-28, 2015

11. Ciuffreda KJ, Kapoor N, Rutner D, Suchoff IB, Han ME, Craig S: Occurrence of oculomotor dysfunctions in acquired brain injury: a retrospective analysis. Optometry 78:155161,2007

12. Corbetta M, Akbudak E, Conturo TE, Snyder AZ, Ollinger JM, Drury HA, et al: A common network of functional areas for attention and eye movements. Neuron 21:761-773, 1998

13. Crevits L, Hanse MC, Tummers P, Van Maele G: Antisaccades and remembered saccades in mild traumatic brain injury. J Neurol 247:179-182, 2000

14. Docherty CL, Valovich McLeod TC, Shultz SJ: Postural control deficits in participants with functional ankle instability as measured by the balance error scoring system. Clin J Sport Med 16:203-208, 2006

15. Dziemianowicz MS, Kirschen MP, Pukenas BA, Laudano E, Balcer LJ, Galetta SL: Sports-related concussion testing. Curr Neurol Neurosci Rep 12:547-559, 2012

16. Finnoff JT, Peterson VJ, Hollman JH, Smith J: Intrarater and interrater reliability of the Balance Error Scoring System (BESS). PM R 1:50-54, 2009

17. Galetta KM, Barrett J, Allen M, Madda F, Delicata D, Tennant AT, et al: The King-Devick test as a determinant of head trauma and concussion in boxers and MMA fighters. Neurology 76:1456-1462, 2011

18. Galetta KM, Brandes LE, Maki K, Dziemianowicz MS, Laudano E, Allen M, et al: The King-Devick test and sportsrelated concussion: study of a rapid visual screening tool in a collegiate cohort. J Neurol Sci 309:34-39, 2011

19. Galetta KM, Morganroth J, Moehringer N, Mueller B, Hasanaj L, Webb N, et al: Adding vision to concussion testing: a prospective study of sideline testing in youth and collegiate athletes. J Neuroophthalmol 35:235-241, 2015

20. Galetta MS, Galetta KM, McCrossin J, Wilson JA, Moster S, Galetta SL, et al: Saccades and memory: baseline associations of the King-Devick and SCAT2 SAC tests in professional ice hockey players. J Neurol Sci 328:28-31, 2013

21. Gavett BE, Stern RA, Cantu RC, Nowinski CJ, McKee AC: Mild traumatic brain injury: a risk factor for neurodegeneration. Alzheimers Res Ther 2:18, 2010

22. Ghajar J: Traumatic brain injury. Lancet 356:923-929, 2000

23. Guskiewicz KM, Weaver NL, Padua DA, Garrett WE Jr: Epidemiology of concussion in collegiate and high school football players. Am J Sports Med 28:643-650, 2000

24. Heitger MH, Jones RD, Dalrymple-Alford JC, Frampton CM, Ardagh MW, Anderson TJ: Motor deficits and recovery during the first year following mild closed head injury. Brain Inj 20:807-824, 2006

25. Hunt AW, Mah K, Reed N, Engel L, Keightley M: Oculomotor-based vision assessment in mild traumatic brain injury: a systematic review. J Head Trauma Rehabil 1:1-10, 2015

26. King D, Brughelli M, Hume P, Gissane C: Assessment, management and knowledge of sport-related concussion: systematic review. Sports Med 44:449-471, 2014

27. King D, Clark T, Gissane C: Use of a rapid visual screening tool for the assessment of concussion in amateur rugby league: a pilot study. J Neurol Sci 320:16-21, 2012

28. King LA, Horak FB, Mancini M, Pierce D, Priest KC, Chesnutt J, et al: Instrumenting the balance error scoring system for use with patients reporting persistent balance problems after mild traumatic brain injury. Arch Phys Med Rehabil 95:353-359, 2014

29. Kraus MF, Little DM, Donnell AJ, Reilly JL, Simonian N, Sweeney JA: Oculomotor function in chronic traumatic brain injury. Cogn Behav Neurol 20:170-178, 2007

30. Langlois JA, Rutland-Brown W, Wald MM: The epidemiology and impact of traumatic brain injury: a brief overview. J Head Trauma Rehabil 21:375-378, 2006

31. Leininger BE, Gramling SE, Farrell AD, Kreutzer JS, Peck EA III: Neuropsychological deficits in symptomatic minor head injury patients after concussion and mild concussion. J Neurol Neurosurg Psychiatry 53:293-296, 1990

32. Leong DF, Balcer LJ, Galetta SL, Evans G, Gimre M, Watt D: The King-Devick test for sideline concussion screening in collegiate football. J Optom 8:131-139, 2015

33. Maruta J: Ocular disconjugacy cannot be measured without establishing a solid spatial reference. F1000 Res 4:71, 2015

34. Maruta J, Ghajar J: Detecting eye movement abnormalities from concussion. Prog Neurol Surg 28:226-233, 2014

35. Maruta J, Heaton KJ, Kryskow EM, Maule AL, Ghajar J: Dynamic visuomotor synchronization: quantification of pre- 
dictive timing. Behav Res Methods 45:289-300, 2013

36. Maruta J, Lee SW, Jacobs EF, Ghajar J: A unified science of concussion. Ann N Y Acad Sci 1208:58-66, 2010

37. Maruta J, Suh M, Niogi SN, Mukherjee P, Ghajar J: Visual tracking synchronization as a metric for concussion screening. J Head Trauma Rehabil 25:293-305, 2010

38. McCrory P, Meeuwisse WH, Johnston K, Dvorak J, Aubry M, Molloy M, et al: Consensus Statement on Concussion in Sport: the 3rd International Conference on Concussion in Sport Held in Zurich, November 2008. Br J Sports Med 43:i76-i84, 2009

39. Meehan WP III, d'Hemecourt P, Comstock RD: High school concussions in the 2008-2009 academic year: mechanism, symptoms, and management. Am J Sports Med 38:24052409,2010

40. Mullen SJ, Yücel YH, Cusimano M, Schweizer TA, Oentoro A, Gupta N: Saccadic eye movements in mild traumatic brain injury: a pilot study. Can J Neurol Sci 41:58-65, 2014

41. Munce TA, Dorman JC, Odney TO, Thompson PA, Valentine VD, Bergeron MF: Effects of youth football on selected clinical measures of neurologic function: a pilot study. J Child Neurol 29:1601-1607, 2014

42. Plassman BL, Havlik RJ, Steffens DC, Helms MJ, Newman TN, Drosdick D, et al: Documented head injury in early adulthood and risk of Alzheimer's disease and other dementias. Neurology 55:1158-1166, 2000

43. Poltavski DV, Biberdorf D: Screening for lifetime concussion in athletes: importance of oculomotor measures. Brain Inj 28:475-485, 2014

44. Rutherford WH, Merrett JD, McDonald JR: Symptoms at one year following concussion from minor head injuries. Injury 10:225-230, 1979

45. Saigal R, Berger MS: The long-term effects of repetitive mild head injuries in sports. Neurosurgery 75 (Suppl 4):S149S155, 2014

46. Samadani U: A new tool for monitoring brain function: eye tracking goes beyond assessing attention to measuring central nervous system physiology. Neural Regen Res 10:12311233,2015

47. Samadani U, Farooq S, Ritlop R, Warren F, Reyes M, Lamm E, et al: Detection of third and sixth cranial nerve palsies with a novel method for eye tracking while watching a short film clip. J Neurosurg 122:707-720, 2015

48. Samadani U, Li M, Qian M, Laska E, Ritlop R: Sensitivity and specificity of an eye movement tracking-based biomarker for concussion. Concussion 2015: CNC3, 2015

49. Samadani U, Ritlop R, Reyes M, Nehrbass E, Li M, Lamm E, et al: Eye tracking detects disconjugate eye movements asso- ciated with structural traumatic brain injury and concussion. J Neurotrauma 32:548-556, 2015

50. Schneiders AG, Sullivan SJ, Handcock P, Gray A, McCrory PR: Sports concussion assessment: the effect of exercise on dynamic and static balance. Scand J Med Sci Sports 22:8590, 2012

51. Suh M, Basu S, Kolster R, Sarkar R, McCandliss B, Ghajar $\mathrm{J}$ : Increased oculomotor deficits during target blanking as an indicator of mild traumatic brain injury. Neurosci Lett 410:203-207, 2006

52. Suh M, Kolster R, Sarkar R, McCandliss B, Ghajar J: Deficits in predictive smooth pursuit after mild traumatic brain injury. Neurosci Lett 401:108-113, 2006

53. Torres DM, Galetta KM, Phillips HW, Dziemianowicz EMS, Wilson JA, Dorman ES, et al: Sports-related concussion: Anonymous survey of a collegiate cohort. Neurol Clin Pract 3:279-287, 2013

54. Ventura RE, Jancuska JM, Balcer LJ, Galetta SL: Diagnostic tests for concussion: is vision part of the puzzle? J Neuroophthalmol 35:73-81, 2015

55. Ventura R, Marinides Z, Galetta S, Clugston J, Balcer L: Vision testing is additive to the sideline assessment of sportsrelated concussion. Neurol Clin Pract [epub ahead of print], 2014

56. Wilkins JC, Valovich McLeod TC, Perrin DH, Gansneder BM: Performance on the Balance Error Scoring System decreases after fatigue. J Athl Train 39:156-161, 2004

57. Williamson RW, Gerhardstein D, Cardenas J, Michael DB, Theodore N, Rosseau N: Concussion 101: the current state of concussion education programs. Neurosurgery 75 (Suppl 4):S131-S135, 2014

\section{Disclosures}

Dr. Ghajar is a director at and owns stock in SyncThink.

\section{Author Contributions}

Conception and design: Sussman. Drafting the article: Sussman. Critically revising the article: all authors. Reviewed submitted version of manuscript: all authors. Approved the final version of the manuscript on behalf of all authors: Ghajar.

\section{Correspondence}

Jamshid Ghajar, Department of Neurosurgery, Stanford University School of Medicine, 300 Pasteur Dr., R200, Stanford, CA 94305-5327. email: jghajar@stanford.edu. 